\title{
Myocardial Bridge in Dextrocardia with Situs Inversus
}

\author{
Yoshiyuki Tohno ${ }^{1 *}$, Setsuko Tohno ${ }^{1}$, Pasuk Mahakkanukrauh ${ }^{1,2,3}$, Cho Azuma ${ }^{4}$, Nutcharin \\ Pakdeewong-Ongkana ${ }^{1}$, Patipath Suwannahoy ${ }^{1}$, Ranida Quiggins ${ }^{1}$, Apichat Sinthubua ${ }^{1}$ and Chollada \\ Mahakkanukrauh ${ }^{5}$
}

\author{
${ }^{1}$ Department of Anatomy, Faculty of Medicine, Chiang Mai University, Thailand \\ ${ }^{2}$ Forensic Osteology Center, Faculty of Medicine, Chiang Mai University, Thailand \\ ${ }^{3}$ Excellence in Osteology Research and Training Center (ORTC), Chiang Mai University, Thailand \\ ${ }^{4}$ Department of Anatomy and Cell Biology, Nara Medical University School of Medicine, Japan \\ ${ }^{5}$ Undergraduate Student, Faculty of Medicine, Chiang Mai University, Thailand
}

*Corresponding author: Yoshiyuki Tohno, Visiting Professor, Department of Anatomy, Faculty of Medicine, Chiang Mai University, Chiang Mai 50200, Thailand, Tel: 66-53-935312, Fax: 66-53-935304

\begin{abstract}
It is ambiguous whether the myocardial bridge (MB) over the coronary arteries and their branches is present in or absent from dextrocardia with situs inversus. Two cases of dextrocardia with situs inversus were accidentally found in adult Thai hearts received for researches from the Department of Anatomy, Faculty of Medicine, Chiang Mai University. They were found in 43- and 78-year-old Thai men. One case of dextrocardia with situs inversus was found in gross anatomy practice of Chiang Mai University. It was found in a 62-year-old Thai man. Therefore, the authors investigated whether the MB over the left and right coronary arteries and their branches was present in or absent from three cases of dextrocardia with situs inversus. The MB was found over the distal left coronary artery in one case of dextrocardia with situs inversus.
\end{abstract}

\section{Keywords}

Dextrocardia, Situs inversus, Myocardial bridge, Coronary artery

\section{Introduction}

Dextrocardia with situs inversus is a very rare condition. Its incidence is one or two in 10,000 [1-3]. Usually, the subject with situs inversus viscerum totalis survives enough long life without congenital defects. It is well known that the myocardial bridge (MB) over the coronary arteries and their branches is frequently present in the normal human heart $[4,5]$. However, it is ambiguous whether the $\mathrm{MB}$ over the coronary arteries and their branches is present in or absent from dextrocardia with situs inversus.

Two cases of dextrocardia with situs inversus were accidentally found in about 330 adult Thai hearts received for researches from the Department of Anatomy, Faculty of Medicine, Chiang Mai University from 2001 to 2015. In addition, one case of dextrocardia with situs inversus was found in gross anatomy practice of Chiang Mai University in 2018. Therefore, the authors investigated whether the MB over the coronary arteries and their branches was present in or absent from three cases of dextrocardia with situs inversus. The MB was found over the distal left coronary artery in one case of dextrocardia with situs inversus.

\section{Materials and Methods}

Thai cadavers were treated by injection of a mixture of $26 \%$ methanol, $14 \%$ glycerin, $3 \%$ phenol, $14 \%$ formalin, $0.34 \mathrm{M}$ potassium nitrate, and $14 \mathrm{mM}$ arsenic oxide through the femoral artery [6]. For researches, the authors received about 330 adult Thai hearts from the Department of Anatomy, Faculty of Medicine, Chiang Mai University from 2001 to 2015 . Two cases of dextrocardia with situs inversus were accidentally found within the adult Thai hearts. In addition, one case of dextrocardia with situs inversus was found in gross anatomy practice of Chiang Mai University in 2018. The hearts were preserved in $10 \%$ formalin solution.

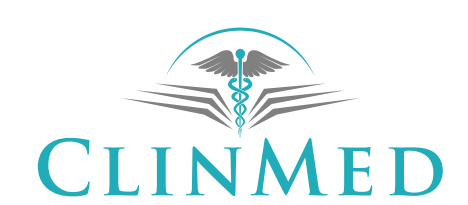

INTERNATIONAL LIBRARY

Citation: Tohno Y, Tohno S, Mahakkanukrauh P, Azuma C, Ongkana PN, et al. (2021) Myocardial Bridge in Dextrocardia with Situs Inversus. Int Arch Vasc Med 3:008. doi.org/10.23937/2690-3164/1710008 Accepted: May 29, 2021: Published: May 31, 2021

Copyright: (C) 2021 Tohno Y, et al. This is an open-access article distributed under the terms of the Creative Commons Attribution License, which permits unrestricted use, distribution, and reproduction in any medium, provided the original author and source are credited. 

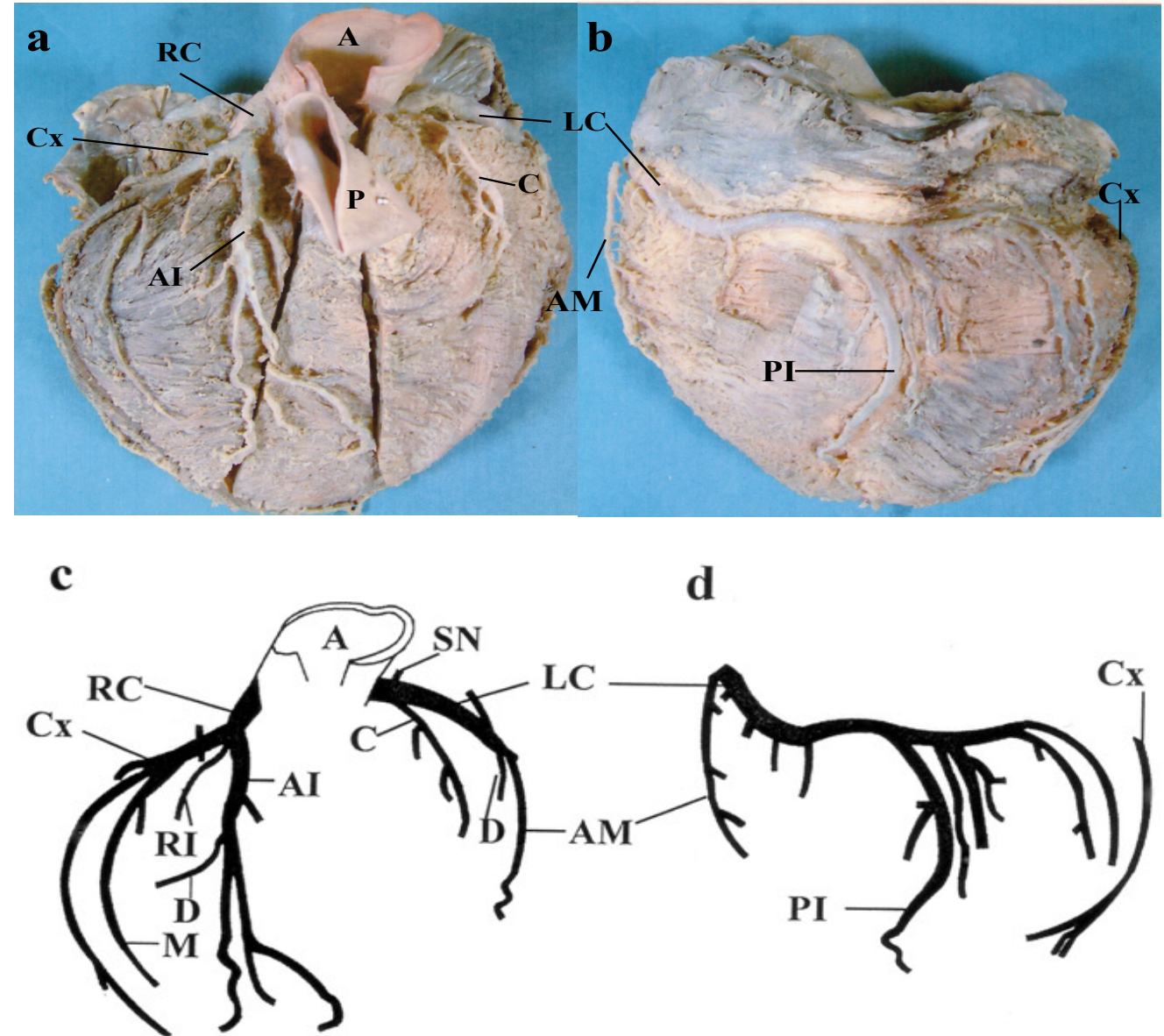

Figure 1: Anterior (a) and posterior (b) views of dextrocardia with situs inversus found in a 43-year-old Thai man and schematic presentation of the left and right coronary arteries and their branches in anterior (c) and posterior (d) views.

A: Ascending Aorta; Al: Anterior Interventricular Artery; AM: Acute Marginal Branch; C: Conus Branch; Cx: Circumflex Artery; D: Diagonal Branch; LC: Left Coronary Artery; M: Marginal Branch; P: Pulmonary Artery; PI: Posterior Interventricular Artery; RC: Right Coronary Artery; SN: Sinoatrial Nodal Artery.

To examine the presence of the MB, fatty tissue was removed from the heart and arterial blood vessels were prepared by careful dissection with special reference to the presence of the MB.

\section{Results}

Three cases of dextrocardia with situs inversus were found in a 43-year-old man (cadaver number 41/47) who died of hepatoma, a 78-year-old man (cadaver number $165 / 56$ ) who died of senility, and a 62 -year-old man (cadaver number 11/60) who died of sepsis.

\section{The first case (cadaver number 41/47)}

The right aortic sinus gave rise to the right coronary artery. The right coronary artery (diameter $7.0 \mathrm{~mm}$ ) was trifurcated into the anterior interventricular artery (or anterior descending artery), the circumflex artery, and the ramus intermedius artery (Figure 1 ). The anterior interventricular artery (diameter $5.1 \mathrm{~mm}$ ) coursed through the anterior interventricular groove and gave off the diagonal branches to the anterior wall of the right ventricle. The circumflex artery (diameter $4.5 \mathrm{~mm}$ ) coursed through the right atrioventricular groove and gave off the marginal branches to the lateral wall of the right ventricle. The small ramus intermedius artery (diameter $1.2 \mathrm{~mm}$ ) distributed the anterior wall of the right ventricle.

The left coronary artery (diameter $5.2 \mathrm{~mm}$ ) arose from the left aortic sinus and coursed through the left atrioventricular groove (Figure 1). The left coronary artery ramified first the sinoatrial nodal artery and secondly the conus branch. The fifth branch was the acute marginal branch and ran along the margin of the left ventricle. The posterior interventricular artery of the left coronary artery coursed downward through the posterior interventricular groove. No MB was found over the left and right coronary arteries and their branches in the first case of dextrocardia with situs inversus.

The coronary circulation of the first case was classified as left dominant, because the posterior interventricular artery was supplied by the left coronary artery [7].

\section{The second case (cadaver number 165/56)}

The right aortic sinus gave rise to the right coronary artery. The right coronary artery (diameter $8.9 \mathrm{~mm}$ ) was trifurcated into the anterior interventricular artery, the circumflex artery, and the ramus intermedius artery 


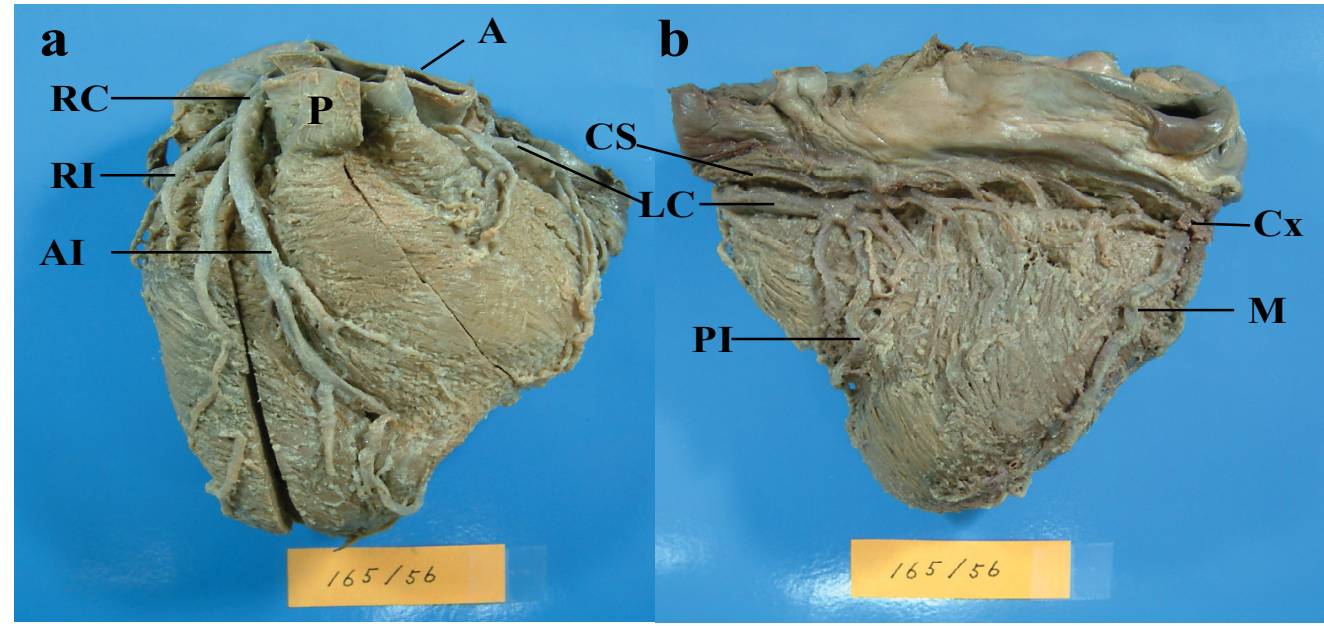

C

d

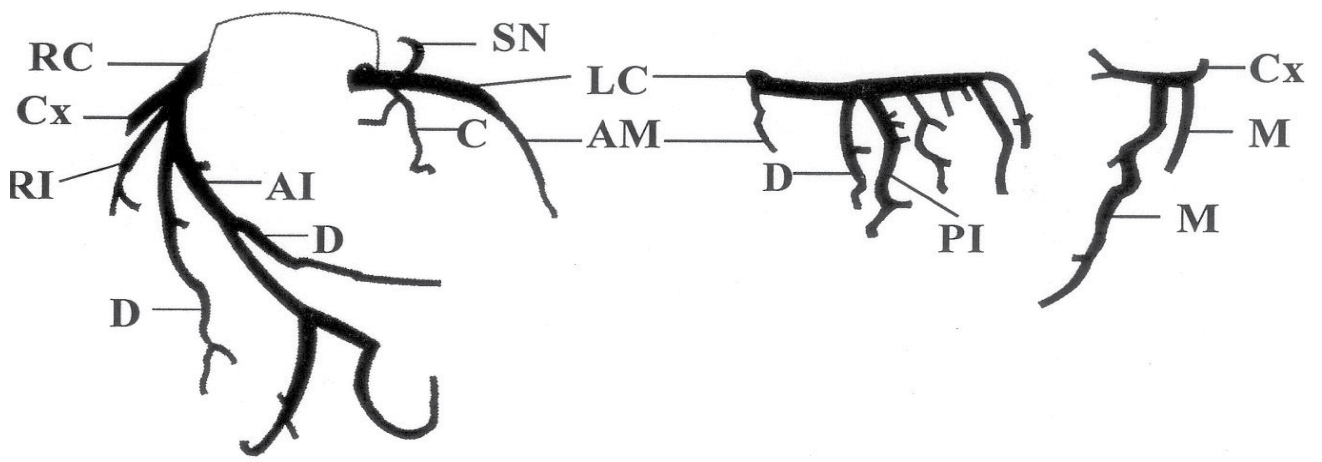

Figure 2: Anterior (a) and posterior (b) views of dextrocardia with situs inversus found in a 78-year-old Thai man and schematic presentation of the left and right coronary arteries and their branches in anterior (c) and posterior (d) views.

A: Ascending Aorta; Al: Anterior Interventricular Artery; AM: Acute Marginal Branch; C: Conus Branch; Cx: Circumflex Artery; CS: Coronary Sinus; D: Diagonal Branch; LC: Left Coronary Artery; M: Marginal Branch; P: Pulmonary Artery; PI: Posterior Interventricular Artery; RC: Right Coronary Artery; SN: Sinoatrial Nodal Artery.

(Figure 2). The anterior interventricular artery (diameter $4.2 \mathrm{~mm}$ ) ran through the anterior interventricular groove and gave off the diagonal branches to the anterior wall of the right ventricle. The circumflex artery (diameter $3.9 \mathrm{~mm}$ ) coursed through the right atrioventricular groove and gave off the marginal branches to the lateral wall of the right ventricle. The ramus intermedius artery (diameter $2.4 \mathrm{~mm}$ ) distributed the anterior wall of the right ventricle.

The left coronary artery (diameter $3.8 \mathrm{~mm}$ ) arose from the left aortic sinus and coursed through the left atrioventricular groove. The left coronary artery ramified both the conus branch and the sinoatrial nodal artery (Figure 2). The acute marginal branch of the left coronary artery ran along the margin of the left ventricle. The posterior interventricular artery of a branch of the left coronary artery coursed downward through the posterior interventricular groove. Some diagonal branches distributed the posterior walls of the left and right ventricles. No MB was found over the left and right coronary arteries and their branches in the second case of dextrocardia with situs inversus.

The coronary circulation of the second case was classified as left dominant, because the posterior interven- tricular artery was supplied by the left coronary artery.

\section{The third case (cadaver number 11/60)}

The right aortic sinus gave rise to the right coronary artery. The right coronary artery (diameter $5.0 \mathrm{~mm}$ ) was trifurcated into the anterior interventricular artery, the circumflex artery, and the ramus intermedius artery (Figure 3). The anterior interventricular artery (diameter $4.4 \mathrm{~mm}$ ) coursed through the anterior interventricular groove and gave off the diagonal branches to the anterior wall of the right ventricle. The circumflex artery (diameter $4.5 \mathrm{~mm}$ ) coursed through the right atrioventricular groove and the posterior interventricular groove and gave off the marginal branches to the posterior wall of the right ventricle. Thereafter, the circumflex artery gave off the posterior interventricular artery. The ramus intermedius artery (diameter $2.5 \mathrm{~mm}$ ) distributed the anterior wall of the right ventricle.

The left coronary artery (diameter $3.5 \mathrm{~mm}$ ) arose from the left aortic sinus and coursed through the left atrioventricular groove (Figure 3). The left coronary artery ramified first the conus branch and secondly the acute marginal branch. The acute marginal branch ran along the margin of the left ventricle. The left coronary 

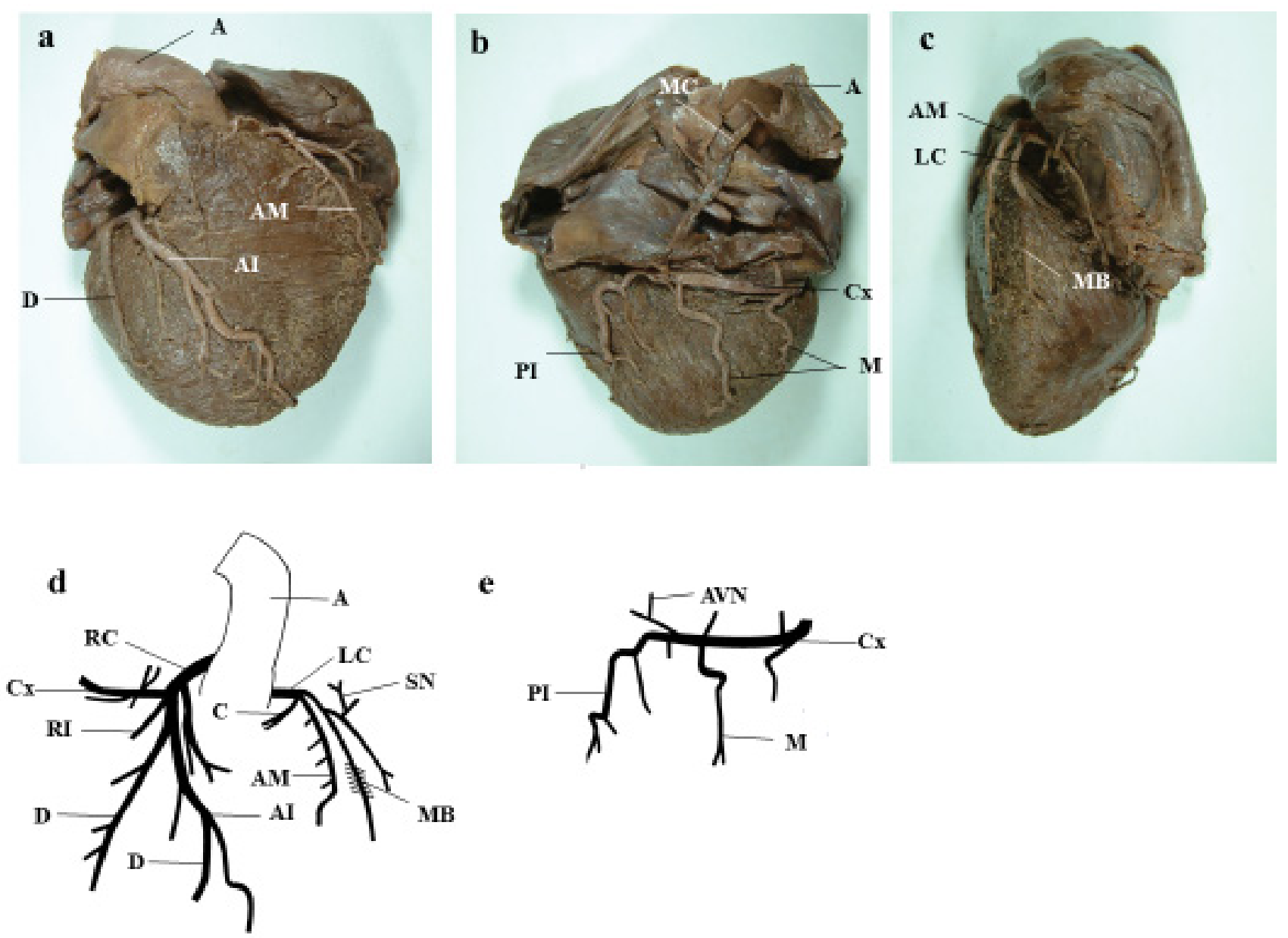

e

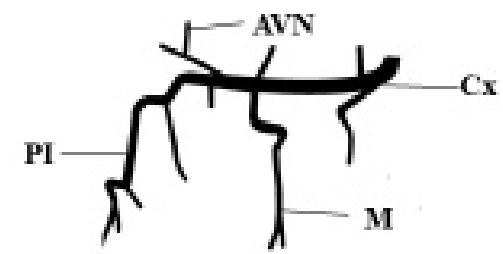

Figure 3: Anterior (a), posterior (b), and left lateral (c) views of dextrocardia with situs inversus found in a 62-year-old Thai man and schematic presentation of the left and right coronary arteries and their branches in anterior (d) and posterior (e) views.

A: Ascending Aorta; Al: Anterior Interventricular Artery; AM: Acute Marginal Branch; AVN: Atrioventricular Nodal Artery; C: Conus Branch; Cx: Circumflex Artery; D: Diagonal Branch; LC: Left Coronary Artery; M: Marginal Branch; MC: Median Cardiac Vein; P: Pulmonary Artery; PI: Posterior Interventricular Artery; RC: Right Coronary Artery; SN: Sinoatrial Nodal Artery.

artery ramified the sinoatrial nodal artery. The myocardial bridge $(1.4 \mathrm{~cm}$ in length) was found over the distal left coronary artery (Figure 3c). The angle between the directions of the myocardial bundles and of the left coronary artery was about $60^{\circ}$.

The coronary circulation of the third case was classified as right dominant, because the posterior interventricular artery was supplied by the right coronary artery.

In three cases, the left and right coronary arteries formed a mirror picture of the normal pattern. The MB was found over the distal left coronary artery in the third case.

\section{Discussion}

There are pathological reports $[2,8,9]$ on dextrocardia with situs inversus. Merklin, et al. [2] examined pathologically 111 cases of situs inversus viscerum and reported that a high number of patients with situs inversus totalis were afflicted with cardiac defects such as tetralogy of Fallot, interatrial septal defect, interventricular septal defect, and so on. Hynes, et al. [9] studied clinically and pathologically the coronary arteries in six cases of dextrocardia with situs inversus, ranging in age from 33 to 72 years and reported that severe atherosclerotic lesions were seen in almost all of the anterior interventricular artery. However, as it is recognized that the $\mathrm{MB}$ is an anatomical variant, they did not examine whether there are the presence or absence of the $M B$ in dextrocardia with situs inversus $[2,8,9]$.

There are a few reports $[10,11]$ on the coronary arteries of dextrocardia with situs inversus. Mano, et al. [10] reported one case of situs inversus viscerum totalis who was a 78-year-old Japanese man. Coronary vessel morphology was clearly inverted. The right-side left coronary artery divided into the anterior interventricular and the circumflex arteries. Conversely, the left-side right coronary artery ran dorsally and rightward along the atrioventricular groove to the crux and gave off the posterior interventricular artery. Roongruangchai, et al. [11] reported one case of situs inversus viscerum totalis who was an 87-year-old Thai woman. The right coronary artery divided into the anterior interventricular and the circumflex arteries. The left coronary artery ran along the left atrioventricular groove and gave off the posterior interventricular artery. The coronary circulation of two cases was classified as left dominant, 
because the posterior interventricular artery was supplied by the left coronary artery. The present study revealed that the coronary circulation of two cases was classified as left dominant, whereas it of one case was classified as right dominant. In the two reports [10,11], the authors did not describe about the presence or absence of the MB over the left and right coronary arteries and their branches. Based on their figures, however, we presumed that the MB was absent from the coronary arteries and their branches in two cases of dextrocardia with situs inversus reported by Mano, et al. [10] and Roongruangchai, et al. [11].

The authors [12] experienced one case of situs inversus viscerum totalis found in a 95-year-old Japanese woman in gross anatomy practice of Nara Medical University in 1982. However, the authors did not pay a special attention to the MB. Therefore, it is ambiguous whether the MB was present in or absent from the case of dextrocardia with situs inversus.

There are numerous clinical reports (for example, Murtuza, et al. [13]; Kuwata, et al. [14]) on coronary artery bypass grafting in patients with dextrocardia and situs inversus totalis. To our knowledge, the authors did not describe about the presence or absence of the MB in dextrocardia with situs inversus in these reports.

Regarding the incidence of the $\mathrm{MB}$ in the normal heart, the MB was found in 23 to $60 \%$ in autopsy series $[4,5]$. By coronary angiography and computed tomography, the MB was found in 0.5 to $58 \%$ of the cases $[4,5]$. The authors [15] recently investigated the MB by dissection using 84 adult Thai hearts and found that the MBs were present in $39(46.4 \%)$ of 84 hearts with a total of $44 \mathrm{MBs}$. It should be noted that 37 out of $44 \mathrm{MBs}$ were located in the middle one third of the anterior interventricular artery. Similar findings were reported by Mohlenkamp, et al. [4] and Ishikawa, et al. [5]. The present study revealed that the $\mathrm{MB}$ was present over the distal left coronary artery in one case of dextrocardia with situs inversus, but it was not present over the anterior interventricular artery.

There are a few reports $[16,17]$ on the mechanism of occurrence of the MB. Tao, et al. [16] reported that the $\mathrm{MB}$ resulted from the gradient of hypoxia inducible factor- $1 \alpha$. The authors take an interest in the mechanism of occurrence of the MB. Therefore, it is worthy of note to examine whether there is a significant difference in the location and the incidence of the MB between normal heart and dextrocardia.

\section{Conclusion}

The authors investigated whether the MB over the left and right coronary arteries and their branches was present in or absent from three cases of dextrocardia with situs inversus. It was found that the MB was present in the distal left coronary artery in one case of dextrocardia with situs inversus.

\section{Conflict of Interest}

The authors disclose no financial or personal relationships. The authors declare no conflict of interest.

\section{References}

1. Blegen HM (1949) Surgery in situs inversus. Ann Surg 129: 244-259.

2. Merklin RJ, Varano NR (1963) Situs inversus and cardiac defects. A study of 111 cases of reversed asymmetry. J Thorac Cardiovasc Surg 45: 334-342.

3. Campbell M, Deuchar DC (1967) Absent inferior vena cava, symmetric liver, splenic agenesis, and situs inversus, and their embryology. Brit Heart J 29: 268-275.

4. Mohlenkamp S, Hort W, Ge J, Erbel R (2002) Update on myocardial bridging. Circulation 106: 2616-2622.

5. Ishikawa Y, Kawawa Y, Kohda E, Shimada K, Ishii T (2011) Significance of the anatomical properties of a myocardial bridge in coronary heart disease. Circulation J 75: 1559-1566.

6. Tohno Y, Tohno S, Mahakkanukrauh P, Vaidhayakarn P, Somsarp V, et al. (2001) Simultaneous accumulation of magnesium with calcium and phosphorus in aorta and iliac arteries of Thai. Biol Trace Elem Res 84: 19-35.

7. Allwork SP (1987) The applied anatomy of the arterial blood supply to the heart in man. J Anat 153: 1-16.

8. Garg N, Agarwal BL, Modi N, Radhakrishnan S, Sinha N (2003) Dextrocardia: An analysis of cardiac structures in 125 patients. Int J Cardiol 88: 143-155.

9. Hynes KM, Gau GT, Titus JL (1973) Coronary heart disease in situs inversus totalis. Am J Cardiol 31: 666-669.

10. Mano Y, Adachi N, Murakami G, Yokoyama T, Dodo $Y$ (2006) Human situs inversus of the thoracoabdominal structures. Anat Sci Int 81: 7-20.

11. Roongruangchai J, Narongsak W, Plakornkul V, Viravud Y, Sripaoraya K, et al. (2012) Situs inversus totalis and ultrastructure of respiratory cilia: Report of a cadaveric case. $\mathrm{J}$ Med Assoc Thai 95: 132-138.

12. Tohno $\mathrm{Y}$, Tohno $\mathrm{S}$, Matsumoto $\mathrm{H}$, Nishimori $\mathrm{K}$, Noda $\mathrm{Y}$, et al. (1983) A case of situs inversus viscerum totalis observed in a 95-year-old female. Kaibogaku Zasshi 58: 606-613.

13. Murtuza B, Gupta P, Goli G, Lall KS (2010) Coronary revascularization in adult with dextrocardia: Surgical implications of the anatomic variants. Texas Heart Inst J 37: 633-640.

14. Kuwata $T$, Kawata $T$, Ueda $H$, Sakaguchi $H$, Nagasaka $S$, et al. (2004) Off-pump quintuple coronary artery bypass grafting for situs inversus totalis. Jap J Thorac Cardiovasc Surg 52: 473-475.

15. Tohno $\mathrm{Y}$, Tohno S, Minami T, Pakdeewong-Ongkana N, Suwannahoy $P$, et al. (2015) Different accumulation of elements in proximal and distal parts of the left anterior descending artery beneath the myocardial bridge. Biol Trace Elem Res 171: 17-25.

16. Tao J, Doughman Y, Yang K, Ramirez-Bergeron D, Watanabe $H$ (2013) Epicardial HIF signaling regulates vascular precursor cell invasion into the myocardium. Dev Biol 376: 136-149.

17. Watanabe Y, Arakawa T, Kageyama I, Aizawa Y, Kumaki K, et al. (2016) Gross anatomical study on the human myocardial bridges with special reference to the spatial relationship among coronary arteries, cardiac veins, and autonomic nerves. Clin Anat 29: 333-341. 